\title{
BEHAVIOR OF SOLUTIONS OF A NONLINEAR IMPULSIVE SYSTEM WITH PIECEWISE CONSTANT MIXED ARGUMENTS
}

\author{
MEHTAP LAFCI BÜYÜKKAHRAMAN, GIZEM S. OZTEPE, \\ AND HÜSEYIN BEREKETOĞLU
}

Received 14 February, 2020

\begin{abstract}
In this paper, we consider a nonlinear impulsive differential equation system with piecewise constant mixed arguments and prove the existence and uniqueness of a solution. Moreover, we obtain sufficient conditions for oscillation of the solution and give an example for it. Finally, we show that the solution of the modified version of this equation is $k$ periodic.
\end{abstract}

2010 Mathematics Subject Classification: 34C25; 34K11; 34K45

Keywords: impulsive differential equation, piecewise constant argument, oscillation, periodicity

\section{INTRODUCTION}

There has been increasing interest in the applications of various neural networks in many areas, such as signal processing, image processing, pattern recognition, fault diagnosis, associative memory, and combinatorial optimization ([8], [9], [11], [13], [16]).

In addition, piecewise constant systems proposed by Busenberg and Cooke ([3]) exist in widely expanded areas such as biomedicine, chemistry, mechanical engineering, physics, etc. Such systems are described as a combination of continuous and discrete (hybrid dynamical systems) and so combine properties of both differential and difference equations. Moreover, cellular neural networks with piecewise constant argument have been investigated by some authors ([7], [10], [14], [15]) and also the qualitative analysis of differential equations with piecewise constant arguments of mixed type has been considered by some authors ([2], [5], [6]).

On the other hand, in the real world, many evolutionary processes are characterized by sudden changes at certain times. These changes are said to be impulsive phenomena that take place in many areas, such as physics, chemistry, population dynamics, optimal control, etc.

Most neural networks can be classified into two categories, either continuous or discrete. However, they exhibit characteristics both continuous and discrete styles in many real world systems and natural processes. So, the impulsive differential 
equations with piecewise constant argument which are named by Wiener ([15]) in 1993 have been important. Recently, impulsive cellular neural networks models with piecewise constant argument have been studied in the papers ([1], [4], [12]).

Abbas and Xia [1] discussed the existence and uniqueness of almost automorphic solutions of the following impulsive model of neural network with piecewise constant argument. These kinds of solutions are more general than periodic and almost periodic solutions. They gave several sufficient conditions for the exponential and global attractivity of the solution.

$$
\begin{aligned}
\frac{d x_{i}(t)}{d t} & =-a_{i}(t) x_{i}(t)+\sum_{j=1}^{m} b_{i j}(t) f_{j}\left(x_{j}(t)\right)+\sum_{j=1}^{m} c_{i j}(t) g_{j}\left(x_{j}\left(2\left[\frac{t+1}{2}\right]\right)\right) \\
& +I_{i}(t), \\
\left.\triangle x_{i}\right|_{t=\tau_{k}} & =J_{k}\left(x_{i}\left(\tau_{k}^{-}\right)\right), \quad i=1,2, \ldots, n, k \in \mathbb{N}, \\
\triangle\left(x_{i}\left(\tau_{k}\right)\right) & =x_{i}\left(\tau_{k}\right)-x_{i}\left(\tau_{k}^{-}\right), x_{i}\left(\tau_{k}^{-}\right)=\lim _{h \rightarrow 0^{-}} x_{i}\left(\tau_{k}+h\right),
\end{aligned}
$$

where $\tau_{k}=2 k-1, \tau_{k}^{-}=2 k-1^{-}$.

Chiu [4] introduced the following impulsive cellular neural network models with piecewise alternately advanced and retarded argument. Some sufficient conditions were established for the existence and global exponential stability of a unique periodic solution.

$$
\begin{aligned}
& \frac{d x_{i}(t)}{d t}=-a_{i}(t) x_{i}(t)+\left\{\sum_{j=1}^{m} b_{i j}(t) f_{j}\left(x_{j}(t)\right)+c_{i j}(t) g_{j}\left(x_{j}\left(2\left[\frac{t+1}{2}\right]\right)\right)\right\}+d_{i}(t), \\
& t \neq 2 k-1, \\
&\left.\triangle x_{i}\right|_{t=2 k-1}=J_{k}\left(x_{i}\left(2 k-1^{-}\right)\right), i=1,2, \ldots, n, k \in \mathbb{N} .
\end{aligned}
$$

Karakoc et al. [12] proved the existence and uniqueness of a solution of the following nonlinear impulsive differential equation system with piecewise constant argument and obtained sufficient conditions for the oscillation of the solution.

$$
\begin{aligned}
x^{\prime}(t) & =-a(t) x(t)-x([t-1]) f(y([t]))+h_{1}(x([t])), \\
y^{\prime}(t) & =-b(t) y(t)-y([t-1]) g(x([t]))+h_{2}(y([t])), t \neq n \in \mathbb{Z}^{+}, t>0, \\
\triangle x(n) & =x\left(n^{+}\right)-x\left(n^{-}\right)=c_{n} x(n), \\
\triangle y(n) & =y\left(n^{+}\right)-y\left(n^{-}\right)=d_{n} y(n), n \in \mathbb{Z}^{+} .
\end{aligned}
$$

In this paper, we consider the following nonlinear impulsive differential equation system with piecewise constant mixed arguments:

$$
\begin{array}{r}
x^{\prime}(t)+a(t) x(t)+x([t-1]) f_{1}(y([t]))+x([t]) f_{2}(y([t]))+x([t+1]) f_{3}(y([t])) \\
-h_{1}(x([t]))=0,
\end{array}
$$




$$
\begin{array}{r}
y^{\prime}(t)+b(t) y(t)+y([t-1]) g_{1}(x([t]))+y([t]) g_{2}(x([t]))+y([t+1]) g_{3}(x([t])) \\
-h_{2}(y([t]))=0, \\
t \neq n \in \mathbb{Z}^{+}, t>0
\end{array}
$$

with the impulse conditions

$$
\begin{aligned}
& \triangle x(n)=\alpha_{n} x(n)+\beta_{n}, \\
& \triangle y(n)=\gamma_{n} y(n)+\delta_{n}, t=n \in \mathbb{Z}^{+},
\end{aligned}
$$

and the initial conditions

$$
x(-1)=x_{-1}, \quad x(0)=x_{0}, \quad y(-1)=y_{-1}, \quad y(0)=y_{0},
$$

where $a, b:(0, \infty) \rightarrow \mathbb{R}$ are continuous functions, $f_{i}, g_{i}, h_{j} \in C(\mathbb{R}, \mathbb{R}), i=1,2,3, j=$ 1,2. $\alpha_{n}, \beta_{n}, \gamma_{n}$, and $\delta_{n}$ are sequences of real numbers such that $\alpha_{n} \neq 1, \gamma_{n} \neq 1$ for all $n \geq 1 . \triangle u(n)=u\left(n^{+}\right)-u\left(n^{-}\right), u\left(n^{+}\right)=\lim _{t \rightarrow n^{+}} u(t), u\left(n^{-}\right)=\lim _{t \rightarrow n^{-}} u(t)$, [.] denotes the greatest integer functions, and $x_{-1}, x_{0}, y_{-1}, y_{0}$ are given real numbers.

\section{EXISTENCE OF SOLUTIONS}

Definition 1. A pair of functions $(x(t), y(t))$ is said to be a solution of (1.1)-(1.2) if it satisfies the following conditions:

(i) $x: \mathbb{R}^{+} \cup\{-1\} \rightarrow \mathbb{R}$ and $y: \mathbb{R}^{+} \cup\{-1\} \rightarrow \mathbb{R}$ are continuous with a possible exception at the points $[t] \in[0, \infty)$,

(ii) $x(t)$ and $y(t)$ are right continuous and have left-hand limits at the points $[t] \in$ $[0, \infty)$,

(iii) $x(t)$ and $y(t)$ are differentiable and satisfy (1.1) for any $t \in \mathbb{R}^{+}$with a possible exception at the points $[t] \in[0, \infty)$ where one-sided derivatives exist,

(iv) $(x(n), y(n))$ satisfies (1.2) for $n \in \mathbb{Z}^{+}$.

Theorem 1. If $\alpha_{n} \neq 1, \gamma_{n} \neq 1$ for all $n \geq 1$ then the initial value problem (1.1)(1.3) has a unique solution $(x(t), y(t))$ on $\{-1\} \cup[0, \infty)$, which can be formulated on the interval $n \leq t<n+1, n \in \mathbb{N}=0,1,2, \ldots$, as

$$
\begin{aligned}
x_{n}(t) & =e^{-\int_{n}^{t} a(u) d u}\left\{x(n)+\left[-x(n-1) f_{1}(y(n))-x(n) f_{2}(y(n))-x(n+1) f_{3}(y(n))\right.\right. \\
& \left.\left.+h_{1}(x(n))\right] \int_{n}^{t} e^{\int_{n}^{s} a(u) d u} d s\right\}, \\
y_{n}(t) & =e^{-\int_{n}^{t} b(u) d u}\left\{y(n)+\left[-y(n-1) g_{1}(x(n))-y(n) g_{2}(x(n))-y(n+1) g_{3}(x(n))\right.\right. \\
& \left.\left.+h_{2}(y(n))\right] \int_{n}^{t} e^{\int_{n}^{s} b(u) d u} d s\right\},
\end{aligned}
$$

where $(x(n), y(n))$ is the unique solution of the difference equations system

$$
x(n+1)=\frac{1}{1-\alpha_{n+1}}\left\{e ^ { - \int _ { n } ^ { n + 1 } a ( u ) d u } \left\{x(n)+\left[-x(n-1) f_{1}(y(n))-x(n) f_{2}(y(n))\right.\right.\right.
$$




$$
\begin{aligned}
& \left.\left.\left.-x(n+1) f_{3}(y(n))+h_{1}(x(n))\right] \int_{n}^{n+1} e^{\int_{n}^{s} a(u) d u} d s\right\}+\beta_{n+1}\right\} \\
y(n+1) & =\frac{1}{1-\gamma_{n+1}}\left\{e ^ { - \int _ { n } ^ { n + 1 } b ( u ) d u } \left\{y(n)+\left[-y(n-1) g_{1}(x(n))-y(n) g_{2}(x(n))\right.\right.\right. \\
& \left.\left.\left.-y(n+1) g_{3}(x(n))+h_{2}(y(n))\right] \int_{n}^{n+1} e^{\int_{n}^{s} b(u) d u} d s\right\}+\delta_{n+1}\right\}
\end{aligned}
$$

for $n \geq 0$ with the initial conditions (1.3).

Proof. Let $(x(n), y(n)) \equiv(x(t), y(t))$ be a solution of (1.1)-(1.2) on the interval $n \leq t<n+1$. So, the system (1.1) can be written as

$$
\begin{array}{r}
x^{\prime}(t)+a(t) x(t)=-x(n-1) f_{1}(y(n))-x(n) f_{2}(y(n))-x(n+1) f_{3}(y(n))+h_{1}(x(n)), \\
y^{\prime}(t)+b(t) y(t)=-y(n-1) g_{1}(x(n))-y(n) g_{2}(x(n))-y(n+1) g_{3}(x(n))+h_{2}(y(n)), \\
n \leq t<n+1 .
\end{array}
$$

(2.3) consists of two linear differential equations which can be solved separately. Let us solve the first equation in system (2.3), the second one will be solved similarly. Multiplying both sides of the first equation in system (2.3) with $\lambda=e^{\int_{n}^{t} a(u) d u}$ and integrating both sides from $n$ to $t$

$$
\begin{aligned}
e^{\int_{n}^{t} a(u) d u} x(t)-x(n) & =\int_{n}^{t} e^{\int_{n}^{s} a(u) d u}\left[-x(n-1) f_{1}(y(n))-x(n) f_{2}(y(n))\right. \\
& \left.-x(n+1) f_{3}(y(n))+h_{1}(x(n))\right] d s .
\end{aligned}
$$

Hence,

$$
\begin{aligned}
x_{n}(t) & =e^{-\int_{n}^{t} a(u) d u}\left\{x(n)+\left[-x(n-1) f_{1}(y(n))-x(n) f_{2}(y(n))\right.\right. \\
& \left.\left.-x(n+1) f_{3}(y(n))+h_{1}(x(n))\right] \int_{n}^{t} e^{\int_{n}^{s} a(u) d u} d s\right\}, \\
y_{n}(t) & =e^{-\int_{n}^{t} b(u) d u}\left\{y(n)+\left[-y(n-1) g_{1}(x(n))-y(n) g_{2}(x(n))\right.\right. \\
& \left.\left.-y(n+1) g_{3}(x(n))+h_{2}(x(n))\right] \int_{n}^{t} e^{\int_{n}^{s} b(u) d u} d s\right\} .
\end{aligned}
$$

On the other hand, for $n-1 \leq t<n$, we have

$$
\begin{aligned}
x_{n-1}(t) & =e^{-\int_{n-1}^{t} a(u) d u}\left\{x(n-1)+\left[-x(n-2) f_{1}(y(n-1))-x(n-1) f_{2}(y(n-1))\right.\right. \\
& \left.\left.-x(n) f_{3}(y(n-1))+h_{1}(x(n-1))\right] \int_{n-1}^{t} e^{\int_{n-1}^{s} a(u) d u} d s\right\}, \\
y_{n-1}(t) & =e^{-\int_{n-1}^{t} b(u) d u}\left\{y(n-1)+\left[-y(n-2) g_{1}(x(n-1))-y(n-1) g_{2}(x(n-1))\right.\right.
\end{aligned}
$$




$$
\left.\left.-y(n) g_{3}(x(n-1))+h_{2}(x(n-1))\right] \int_{n-1}^{t} e^{\int_{n-1}^{s} b(u) d u} d s\right\} .
$$

From impulse conditions

$$
\triangle x(n)=x\left(n^{+}\right)-x\left(n^{-}\right)=\alpha_{n} x(n)+\beta_{n} .
$$

Since the solution is right continuous,

$$
x\left(n^{-}\right)=\left(1-\alpha_{n}\right) x(n)-\beta_{n} \Longrightarrow x_{n-1}(n)=\left(1-\alpha_{n}\right) x_{n}(n)-\beta_{n} .
$$

Taking $t=n$ at (2.4), (2.5) and substituting (2.6),

$$
\begin{aligned}
\left(1-\alpha_{n}\right) x(n) & =e^{-\int_{n-1}^{n} a(u) d u}\left\{x(n-1)+\left[-x(n-2) f_{1}(y(n-1))-x(n-1) f_{2}(y(n-1))\right.\right. \\
& \left.\left.-x(n) f_{3}(y(n-1))+h_{1}(x(n-1))\right] \int_{n-1}^{n} e^{\int_{n-1}^{s} a(u) d u} d s\right\}+\beta_{n} .
\end{aligned}
$$

Dividing both sides with $\left(1-\alpha_{n}\right)$ and taking $n \rightarrow n+1$, and following the same procedure above for the other equation's solution $y(t)$, we get the following difference system

$$
\begin{aligned}
x(n+1) & =\frac{1}{1-\alpha_{n+1}}\left\{e ^ { - \int _ { n } ^ { n + 1 } a ( u ) d u } \left\{x(n)+\left[-x(n-1) f_{1}(y(n))-x(n) f_{2}(y(n))\right.\right.\right. \\
& \left.\left.\left.-x(n+1) f_{3}(y(n))+h_{1}(x(n))\right] \int_{n}^{n+1} e^{\int_{n}^{s} a(u) d u} d s\right\}+\beta_{n+1}\right\} \\
y(n+1) & =\frac{1}{1-\gamma_{n+1}}\left\{e ^ { - \int _ { n } ^ { n + 1 } b ( u ) d u } \left\{y(n)+\left[-y(n-1) g_{1}(x(n))-y(n) g_{2}(x(n))\right.\right.\right. \\
& \left.\left.\left.-y(n+1) g_{3}(x(n))+h_{2}(y(n))\right] \int_{n}^{n+1} e^{\int_{n}^{s} b(u) d u} d s\right\}+\delta_{n+1}\right\}
\end{aligned}
$$

for $n \geq 0$. Take $n=0$, then

$$
\begin{aligned}
x(1) & =\frac{1}{1-\alpha_{1}}\left\{e ^ { - \int _ { 0 } ^ { 1 } a ( u ) d u } \left\{x(0)+\left[-x(-1) f_{1}(y(0))-x(0) f_{2}(y(0))\right.\right.\right. \\
& \left.\left.\left.-x(1) f_{3}(y(0))+h_{1}(x(0))\right] \int_{0}^{1} e^{\int_{0}^{s} a(u) d u} d s\right\}+\beta_{1}\right\} .
\end{aligned}
$$

Since we know $x(-1), x(0), y(0)$, we can solve $x(1)$ uniquely and the same way can be applied for $y(1)$ also. So, step by step we can find solutions. Hence, the solution of the difference system and also the solution of the system (1.1)-(1.2) is unique.

Remark 1. In the case of $a(t) \equiv a, b(t) \equiv b$ in the system (1.1), the solution (2.1) of (1.1)-(1.2) and the difference equations system (2.2) are reduced to the following forms, respectively:

$$
x_{n}(t)=e^{-a(t-n)}\left\{x(n)+\left[-x(n-1) f_{1}(y(n))-x(n) f_{2}(y(n))-x(n+1) f_{3}(y(n))\right.\right.
$$




$$
\begin{aligned}
& \left.\left.+h_{1}(x(n))\right]\left(\frac{e^{a(t-n)}-1}{a}\right)\right\}, \\
y_{n}(t) & =e^{-b(t-n)}\left\{y(n)+\left[-y(n-1) g_{1}(x(n))-y(n) g_{2}(x(n))-y(n+1) g_{3}(x(n))\right.\right. \\
& \left.\left.+h_{2}(y(n))\right]\left(\frac{e^{b(t-n)}-1}{b}\right)\right\}
\end{aligned}
$$

and

$$
\begin{aligned}
x(n+1) & =\frac{1}{1-\alpha_{n+1}}\left\{e ^ { - a } \left\{x(n)+\left[-x(n-1) f_{1}(y(n))-x(n) f_{2}(y(n))\right.\right.\right. \\
& \left.\left.\left.-x(n+1) f_{3}(y(n))+h_{1}(x(n))\right]\left(\frac{e^{a}-1}{a}\right)\right\}+\beta_{n+1}\right\}, \\
y(n+1) & =\frac{1}{1-\gamma_{n+1}}\left\{e ^ { - b } \left\{y(n)+\left[-y(n-1) g_{1}(x(n))-y(n) g_{2}(x(n))\right.\right.\right. \\
& \left.\left.\left.-y(n+1) g_{3}(x(n))+h_{2}(y(n))\right]\left(\frac{e^{b}-1}{b}\right)\right\}+\delta_{n+1}\right\} .
\end{aligned}
$$

\section{MAIN RESULTS}

Definition 2. A solution $x(t)$ and $y(t)$ of the first and second equations of system (1.1), respectively are said to be oscillatory if $x(t)$ and $y(t)$ have arbitrarily large zeros. Otherwise, it is said to be nonoscillatory.

Definition 3. The solution of system (1.1)-(1.2) is called oscillatory if $x(t)$ and $y(t)$ are oscillatory.

Definition 4. A solution $x(n)$ (or $y(n)$ ) is said to be oscillatory if it is neither eventually positive nor eventually negative. Otherwise, it is said to be nonoscillatory.

Theorem 2. Assume that there exists $M_{i}>0$ and $N_{j}>0$ such that $f_{i}(u) \geq M_{i}$ and $g_{j}(u) \geq N_{j}, i, j=1,3, f_{2}(u), g_{2}(u)>0$ for all $u \in \mathbb{R}, u h_{1}(u)<0$ and $u h_{2}(u)<0$ for $u \neq 0$, and $\alpha_{n}<1, \gamma_{n}<1$ and $\beta_{n}=0, \delta_{n}=0$ for $n \in \mathbb{Z}^{+}$. If the following conditions are satisfied, then all solutions of system (2.2) are oscillatory:

$$
\begin{array}{r}
\lim _{n \rightarrow \infty} \sup \left[\int_{n}^{n+1}\left(1-\alpha_{n}\right) e^{\int_{n-1}^{s} a(u) d u} d s\right. \\
\left.+M_{3} \int_{n}^{n+1} e^{\int_{n}^{s} a(u) d u} d s \int_{n-1}^{n} e^{\int_{n-1}^{s} a(u) d u} d s\right]>\frac{1}{M_{1}}, \\
\lim _{n \rightarrow \infty} \sup \left[\int_{n}^{n+1}\left(1-\gamma_{n}\right) e^{\int_{n-1}^{s} b(u) d u} d s\right. \\
\left.+N_{3} \int_{n}^{n+1} e^{\int_{n}^{s} b(u) d u} d s \int_{n-1}^{n} e^{\int_{n-1}^{s} b(u) d u} d s\right]>\frac{1}{N_{1}} .
\end{array}
$$


Proof. Let $(x(n), y(n))$ be a solution of difference equation system (2.2). Suppose that $x(n)>0, x(n-1)>0$, and $x(n-2)>0$ for $n>N$, where $N$ is sufficiently large. From the first equation of (2.2), taking $n-1$ in place of $n$, we have

$$
\begin{array}{r}
{\left[\left(1-\alpha_{n}\right) e^{\int_{n-1}^{n} a(u) d u}+f_{3}(y(n-1)) \int_{n-1}^{n} e^{\int_{n-1}^{s} a(u) d u} d s\right] x(n)} \\
=x(n-1)+\left[-x(n-2) f_{1}(y(n-1))-x(n-1) f_{2}(y(n-1))\right. \\
\left.+h_{1}(x(n-1))\right] \int_{n-1}^{n} e^{\int_{n-1}^{s} a(u) d u} d s \\
<x(n-1) .
\end{array}
$$

Hence,

$$
\left[\left(1-\alpha_{n}\right) e^{\int_{n-1}^{n} a(u) d u}+f_{3}(y(n-1)) \int_{n-1}^{n} e^{\int_{n-1}^{s} a(u) d u} d s\right] x(n)<x(n-1) .
$$

Multiplying both sides of this inequality by $-f_{1}(y(n)) \int_{n}^{n+1} e^{\int_{n}^{s} a(u) d u} d s<0$ and adding $x(n)+h_{1}(x(n)) \int_{n}^{n+1} e^{\int_{n}^{s} a(u) d u} d s$ to both sides, we obtain from (2.2) that

$$
\begin{aligned}
& -\left[\left(1-\alpha_{n}\right) e^{\int_{n-1}^{n} a(u) d u}+f_{3}(y(n-1)) \int_{n-1}^{n} e^{\int_{n-1}^{s} a(u) d u} d s\right] x(n) f_{1}(y(n)) \int_{n}^{n+1} e^{\int_{n}^{s} a(u) d u} d s \\
& +x(n)+h_{1}(x(n)) \int_{n}^{n+1} e^{\int_{n}^{s} a(u) d u} d s \\
& >-x(n-1) f_{1}(y(n)) \int_{n}^{n+1} e^{\int_{n}^{s} a(u) d u} d s+x(n)+h_{1}(x(n)) \int_{n}^{n+1} e^{\int_{n}^{s} a(u) d u} d s \\
& >x(n)+\left[-x(n-1) f_{1}(y(n))-x(n) f_{2}(y(n))+h_{1}(x(n))\right] \int_{n}^{n+1} e^{\int_{n}^{s} a(u) d u} d s \\
& =\left[\left(1-\alpha_{n+1}\right) e^{\int_{n}^{n+1} a(u) d u}+f_{3}(y(n)) \int_{n}^{n+1} e^{\int_{n+1}^{s} a(u) d u} d s\right] x(n+1) \\
& >0 .
\end{aligned}
$$

Hence,

$$
\begin{array}{r}
-\left[\left(1-\alpha_{n}\right) e^{\int_{n-1}^{n} a(u) d u}+f_{3}(y(n-1)) \int_{n-1}^{n} e^{\int_{n-1}^{s} a(u) d u} d s\right] x(n) f_{1}(y(n)) \\
\cdot \int_{n}^{n+1} e^{\int_{n}^{s} a(u) d u} d s+x(n)+h_{1}(x(n)) \int_{n}^{n+1} e^{\int_{n}^{s} a(u) d u} d s>0
\end{array}
$$

Since $x(n)>0, n>N$, and $h_{1}(x(n))<0$, and dividing to $x(n)$ to both sides of (3.3) we get

$$
1>\left[\left(1-\alpha_{n}\right) \int_{n}^{n+1} e^{\int_{n-1}^{s} a(u) d u} d s+M_{3} \int_{n-1}^{n} e^{\int_{n-1}^{s} a(u) d u} d s \int_{n}^{n+1} e^{\int_{n}^{s} a(u) d u} d s\right] M_{1} .
$$


So, we have

$$
\begin{aligned}
\frac{1}{M_{1}} & \geq \lim _{n \rightarrow \infty} \sup \left[\left(1-\alpha_{n}\right) \int_{n}^{n+1} e^{\int_{n-1}^{s} a(u) d u} d s\right. \\
& \left.+M_{3} \int_{n-1}^{n} e^{\int_{n-1}^{s} a(u) d u} d s \int_{n}^{n+1} e^{\int_{n}^{s} a(u) d u} d s\right],
\end{aligned}
$$

which contradicts (3.1). If $x(n)<0, x(n-1)<0$, and $x(n-2)<0$ for $n>N$, then we obtain the same contradiction. So, the component $x(n)$ of the solution $(x(n), y(n))$ is oscillatory. Similarly, we can show that the component $y(n)$ is oscillatory under condition (3.2). Hence, the proof is complete.

Corollary 1. Under the hypotheses of Theorem 2, all solutions of system (1.1)(1.3) are oscillatory.

Remark 2. In the case of $a(t) \equiv a, b(t) \equiv b$ in the system (1.1), the conditions (3.1) and (3.2) reduce to the following conditions:

$$
\begin{gathered}
\lim _{n \rightarrow \infty} \sup \left[\left(1-\alpha_{n}\right)\left(\frac{e^{2 a}-e^{a}}{a}\right)+M_{3}\left(\frac{e^{a}-1}{a}\right)^{2}\right]>\frac{1}{M_{1}}, \\
\lim _{n \rightarrow \infty} \sup \left[\left(1-\gamma_{n}\right)\left(\frac{e^{2 b}-e^{b}}{b}\right)+N_{3}\left(\frac{e^{b}-1}{b}\right)^{2}\right]>\frac{1}{N_{1}} .
\end{gathered}
$$

Theorem 3. Assume that there exists $K_{1}, K_{2}>0, M_{i}>0$ and $N_{i}>0$ such that $f_{i}(u) \geq M_{i}$ and $g_{i}(u) \geq N_{i}, i=1,2,3$, for all $u \in \mathbb{R}, u h_{j}(u)<0, j=1,2$ for $u \neq 0$, $\alpha_{n} \leq 1-K_{1}, \gamma_{n} \leq 1-K_{2}$ and $\beta_{n}=0, \delta_{n}=0$ for $n \in \mathbb{N}$. Suppose that the following conditions are satisfied:

$$
\begin{array}{r}
\left(M_{2} \liminf _{n \rightarrow \infty} \int_{n}^{n+1} e^{\int_{n}^{s} a(u) d u} d s-1\right)^{2}<4 M_{1} \liminf _{n \rightarrow \infty} \int_{n}^{n+1} e^{\int_{n}^{s} a(u) d u} d s\left(K_{1} \liminf _{n \rightarrow \infty} e^{\int_{n}^{n+1} a(u) d u}\right. \\
\left.+M_{3} \liminf _{n \rightarrow \infty} \int_{n}^{n+1} e^{\int_{n}^{s} a(u) d u} d s\right)<\infty, \\
(3.4) \\
\left(N_{2} \liminf _{n \rightarrow \infty} \int_{n}^{n+1} e^{\int_{n}^{s} b(u) d u} d s-1\right)^{2}<4 N_{1} \liminf _{n \rightarrow \infty} \int_{n}^{n+1} e^{\int_{n}^{s} b(u) d u} d s\left(K_{2} \liminf _{n \rightarrow \infty} e^{\int_{n}^{n+1} b(u) d u}\right. \\
\left.+N_{3} \liminf _{n \rightarrow \infty} \int_{n}^{n+1} e^{\int_{n}^{s} b(u) d u} d s\right)<\infty,
\end{array}
$$

then all solutions of (2.2) are oscillatory.

Proof. Let $(x(n), y(n))$ be a solution of (2.2). We need to show that under condition (3.4), $x(n)$ is oscillatory. Assume that $x(n)>0, x(n-1)>0$ for $n>N$, where $N$ is sufficiently large. From the first equation of (2.2) we obtain that

$1=\frac{x(n+1)}{x(n)}\left(1-\alpha_{n+1}\right) e^{\int_{n}^{n+1} a(u) d u}$ 


$$
+\left[\frac{x(n-1)}{x(n)} f_{1}(y(n))+f_{2}(y(n))+\frac{x(n+1)}{x(n)} f_{3}(y(n))-\frac{h_{1}(x(n))}{x(n)}\right] \int_{n}^{n+1} e^{\int_{n}^{s} a(u) d u} d s .
$$

Let $v_{n}=\frac{x(n)}{x(n-1)}$. Since $v_{n}>0, \lim _{n \rightarrow \infty}$ inf $v_{n} \geq 0$, we have

$$
1 \geq v_{n+1}\left(1-\alpha_{n+1}\right) e^{\int_{n}^{n+1} a(u) d u}+\left[\frac{1}{v_{n}} M_{1}+M_{2}+v_{n+1} M_{3}\right] \int_{n}^{n+1} e^{\int_{n}^{s} a(u) d u} d s .
$$

So, we need to consider two cases.

Case 1. Let $\liminf _{n \rightarrow \infty} v_{n}=v=+\infty$. Then, from (3.6) we get

$$
\begin{array}{r}
1 \geq \liminf _{n \rightarrow \infty} v_{n+1} \liminf _{n \rightarrow \infty}\left(1-\alpha_{n+1}\right) \liminf _{n \rightarrow \infty} e^{\int_{n}^{n+1} a(u) d u} \\
+M_{1} \liminf _{n \rightarrow \infty} \frac{1}{v_{n}} \liminf _{n \rightarrow \infty} \int_{n}^{n+1} e^{\int_{n}^{s} a(u) d u} d s \\
+M_{2} \liminf _{n \rightarrow \infty} \int_{n}^{n+1} e^{\int_{n}^{s} a(u) d u} d s \\
+M_{3} \liminf _{n \rightarrow \infty} v_{n+1} \liminf _{n \rightarrow \infty} \int_{n}^{n+1} e^{\int_{n}^{s} a(u) d u} d s
\end{array}
$$

which is a contradiction. So, we consider the second case.

Case 2. Let $\liminf _{n \rightarrow \infty} v_{n}=v<\infty$. If the first equation of (2.2) is divided by $x(n-1)$, then we have

$$
\begin{aligned}
\frac{x(n)}{x(n-1)} & =\frac{x(n+1)}{x(n-1)}\left(1-\alpha_{n+1}\right) e^{\int_{n}^{n+1} a(u) d u} \\
& +\left[f_{1}(y(n))+\frac{x(n)}{x(n-1)} f_{2}(y(n))+\frac{x(n+1)}{x(n-1)} f_{3}(y(n))-\frac{h_{1}(x(n))}{x(n-1)}\right] \\
& \cdot \int_{n}^{n+1} e^{\int_{n}^{s} a(u) d u} d s .
\end{aligned}
$$

and then we obtain that

$$
v_{n} \geq v_{n} v_{n+1}\left(1-\alpha_{n+1}\right) e^{\int_{n}^{n+1} a(u) d u}+\left(M_{1}+v_{n} M_{2}+v_{n} v_{n+1} M_{3}\right) \int_{n}^{n+1} e^{\int_{n}^{s} a(u) d u} d s .
$$

Taking the inferior limit on both sides of inequality (3.7), we get

$$
\begin{aligned}
& v \geq v^{2} \liminf _{n \rightarrow \infty}\left(1-\alpha_{n+1}\right) \liminf _{n \rightarrow \infty} e^{\int_{n}^{n+1} a(u) d u}+\left(M_{1}+v M_{2}+v^{2} M_{3}\right) \\
& \cdot \liminf _{n \rightarrow \infty} \int_{n}^{n+1} e^{\int_{n}^{s} a(u) d u} d s .
\end{aligned}
$$


Let $\liminf _{n \rightarrow \infty} e^{\int_{n}^{n+1} a(u) d u}=A$ and $\liminf _{n \rightarrow \infty} \int_{n}^{n+1} e^{\int_{n}^{s} a(u) d u} d s=B$. Then the last inequality can be written as

$$
v \geq v^{2} \liminf _{n \rightarrow \infty}\left(1-\alpha_{n+1}\right) A+\left(M_{1}+v M_{2}+v^{2} M_{3}\right) B .
$$

Now, we consider two subcases: (i) If $\liminf _{n \rightarrow \infty}\left(1-\alpha_{n+1}\right)=\infty$, then we have a contradiction from (3.8). (ii) Assume that $0<K_{1} \leq \liminf _{n \rightarrow \infty}\left(1-\alpha_{n+1}\right)<\infty$. Then from (3.8) we have

$$
\left(K_{1} A+M_{3} B\right) v^{2}+\left(M_{2} B-1\right) v+M_{1} B \leq 0
$$

or

$$
\left(K_{1} A+M_{3} B\right)\left[\left(v+\frac{M_{2} B-1}{2\left(K_{1} A+M_{3} B\right)}\right)^{2}+\frac{-\left(M_{2} B-1\right)^{2}+4 M_{1} B\left(K_{1} A+M_{3} B\right)}{4\left(K_{1} A+M_{3} B\right)^{2}}\right] \leq 0 .
$$

Since $A>0, K_{1}>0, M_{3}>0$, and $B>0$, we have

$$
\frac{-\left(M_{2} B-1\right)^{2}+4 M_{1} B\left(K_{1} A+M_{3} B\right)}{4\left(K_{1} A+M_{3} B\right)^{2}} \leq 0,
$$

which contradicts condition (3.4). In the case of $x(n)<0, x(n-1)<0$ for sufficiently large $n>N$, the proof is similar, and we obtain the same contradiction. On the other hand, if we assume that $y(n)$ is a nonoscillatory sequence, then we have a contradiction to condition (3.5). Hence, $(x(n), y(n))$ is an oscillatory solution of the system (2.2).

Corollary 2. Under the hypothesis of Theorem 3, all solutions of system (1.1)(1.2) are oscillatory.

Remark 3. In the case of $a(t) \equiv a, b(t) \equiv b$ in the system (1.1), the conditions (3.4) and (3.5) reduce to the following conditions:

$$
\begin{gathered}
4 M_{1} \frac{e^{a}-1}{a}\left(K_{1} e^{a}+M_{3}\left(\frac{e^{a}-1}{a}\right)\right)>\left(M_{2}\left(\frac{e^{a}-1}{a}\right)-1\right)^{2}, \\
4 N_{1} \frac{e^{b}-1}{b}\left(K_{2} e^{b}+N_{3}\left(\frac{e^{b}-1}{b}\right)\right)>\left(N_{2}\left(\frac{e^{b}-1}{b}\right)-1\right)^{2} .
\end{gathered}
$$

Theorem 4. The solution of the system in the case of $a(t) \equiv a, b(t) \equiv b, \alpha_{n} \equiv$ $\alpha, \beta_{n} \equiv \beta, \gamma_{n} \equiv \gamma, \delta_{n} \equiv \delta$ in (1.1)-(1.2) is $k$ periodic, $k \in \mathbb{N}-\{0\}$, if and only if

$$
x(0)=x(k), y(0)=y(k), x(-1)=x(k-1), y(-1)=y(k-1),
$$

here $x(n)$ and $y(n)$ are solutions of the equations in (2.2).

Proof. If $x_{n}(t)$ is periodic with period $k$, then

$$
x_{k+n}(t)=x_{n}(t-k), k+n \leq t<k+n+1, n=0,1,2, \ldots
$$


This implies that the equalities (3.9) are true. For the proof of sufficiency case, assume that (3.9) is satisfied. So, for $n=0$, we should see

$$
x_{k}(t)=x_{0}(t-k), k \leq t<k+1 .
$$

Taking $n=0$ in (2.8),

$$
\begin{aligned}
x_{0}(t) & =e^{-a t}\{x(0) \\
& \left.+\left[-x(-1) f_{1}(y(0))-x(0) f_{2}(y(0))-x(1) f_{3}(y(0))+h_{1}(x(0))\right]\left(\frac{e^{a t}-1}{a}\right)\right\}
\end{aligned}
$$

and writing $t=t-k$ in (3.10), then

$$
\begin{aligned}
x_{0}(t-k) & =e^{-a(t-k)}\left\{x(0)+\left[-x(-1) f_{1}(y(0))-x(0) f_{2}(y(0))-x(1) f_{3}(y(0))\right.\right. \\
& \left.\left.+h_{1}(x(0))\right]\left(\frac{e^{a(t-k)}-1}{a}\right)\right\} .
\end{aligned}
$$

Now taking $n=k$ in (2.8),

$$
\begin{aligned}
x_{k}(t) & =e^{-a(t-k)}\left\{x(k)+\left[-x(k-1) f_{1}(y(k))-x(k) f_{2}(y(k))\right.\right. \\
& \left.\left.-x(k+1) f_{3}(y(k))+h_{1}(x(k))\right]\left(\frac{e^{a(t-k)}-1}{a}\right)\right\} .
\end{aligned}
$$

Under the assumptions (3.9), we need to show that $x(1)=x(k+1)$. Taking $t=1$ in (3.10), we get

$$
x_{0}(1)=\frac{e^{-a}\left\{x(0)+\left[-x(-1) f_{1}(y(0))-x(0) f_{2}(y(0))\right]\left(\frac{e^{a}-1}{a}\right)\right\}}{1+e^{-a} f_{3}(y(0))\left(\frac{e^{a}-1}{a}\right)}
$$

and from impulse condition for $n=1, n=k+1$, respectively, we have

$$
\begin{gathered}
x_{0}(1)=(1-\alpha) x_{1}(1)-\beta, \\
x_{k}(k+1)=(1-\alpha) x_{k+1}(k+1)-\beta .
\end{gathered}
$$

Writing $t=k+1$ in (3.11), we obtain

$$
x_{k}(k+1)=\frac{e^{-a}\left\{x(k)+\left[-x(k-1) f_{1}(y(k))-x(k) f_{2}(y(k))+h_{1}(x(k))\right]\left(\frac{e^{a}-1}{a}\right)\right.}{1+e^{-a} f_{3}(y(k))\left(\frac{e^{a}-1}{a}\right)} .
$$

Since $x(0)=x(k), y(0)=y(k), x(-1)=x(k-1), y(-1)=y(k-1)$, we find $x(1)=$ $x(k+1)$. Similarly, it can be shown that $y(1)=y(k+1)$. So, we conclude that

$$
x_{k}(t)=x_{0}(t-k), k \leq t<k+1 .
$$


Now, taking $n=1, t=t-k$ and $n=k+1$ in Eq. (2.8), respectively, then

$$
\begin{aligned}
x_{1}(t-k) & =e^{-a(t-k-1)}\left\{x(0)+\left[-x(-1) f_{1}(y(0))-x(0) f_{2}(y(0))-x(1) f_{3}(y(0))\right.\right. \\
& \left.\left.+h_{1}(x(0))\right]\left(\frac{e^{a(t-k-1)}-1}{a}\right)\right\}, \\
x_{k+1}(t) & =e^{-a(t-k-1)}\left\{x(k+1)+\left[-x(k) f_{1}(y(k+1))-x(k+1) f_{2}(y(k+1))\right.\right. \\
& \left.\left.-x(k+2) f_{3}(y(k+1))+h_{1}(x(k+1))\right]\left(\frac{e^{a(t-k-1)}-1}{a}\right)\right\} .
\end{aligned}
$$

Hence, we find

$$
x_{k+1}(t)=x_{1}(t-k), k+1 \leq t<k+2 .
$$

From (3.9) and $x(1)=x(k+1)$, if we show that $x(2)=x(k+2)$, then we can say

$$
x_{k+1}(t)=x_{1}(t-k), k+1 \leq t<k+2 .
$$

From impulse conditions for $n=2$,

$$
x_{1}(2)=(1-\alpha) x_{2}(2)-\beta .
$$

Take $n=1$ and $t=2$ in (2.8) for $x_{1}(2)$

$$
\begin{array}{r}
e^{-a}\left\{x(1)+\left[-x(0) f_{1}(y(1))-x(1) f_{2}(y(1))-x(1) f_{3}(y(1))+h_{1}(x(1))\right]\left(\frac{e^{a}-1}{a}\right)\right\} \\
=(1-\alpha) x(2)-\beta .
\end{array}
$$

So, we can write $x(2)$ in terms of $x(0), x(1), y(1)$ and similarly for $n=k+2$,

$$
x_{k+1}(k+2)=(1-\alpha) x_{k+2}(k+2) \text {. }
$$

Taking $n=k+1$ and $t=k+2$ in (2.8), then

$$
\begin{array}{r}
e^{-a}\left\{x(k+1)+\left[-x(k) f_{1}(y(k+1))-x(k+1) f_{2}(y(k+1))-x(k+2) f_{3}(y(k+1))\right.\right. \\
\left.\left.+h_{1}(x(k+1))\right]\left(\frac{e^{a}-1}{a}\right)\right\}=(1-\alpha) x(k+2)-\beta .
\end{array}
$$

$x(k+2)$ is written in terms of $x(k), x(k+1)$ and $y(k+1)$. By induction,

$$
x_{k+n}(t)=x_{n}(t-k), k+n \leq t<k+n+1 .
$$

One can show that

$$
y_{k+n}(t)=y_{n}(t-k), k+n \leq t<k+n+1
$$

by using the steps above.

Example 1. We consider the nonlinear impulsive differential equations system with piecewise constant argument and variable coefficient

$$
\begin{array}{r}
x^{\prime}(t)+\frac{3}{t} x(t)+x([t-1])\left(e^{-2 y([t])}+3\right)+x([t])\left(e^{-2 y([t])}+2\right) \\
+x([t+1])\left(e^{-2 y([t])}+1\right)-x([t])=0,
\end{array}
$$




$$
\begin{array}{r}
y^{\prime}(t)+\frac{3 t^{2}}{t^{3}+1} y(t)+y([t-1])\left(x^{2}([t])+3\right)+y([t])\left(x^{2}([t])+2\right) \\
+y([t+1])\left(x^{2}([t])+1\right)-y([t])=0, \\
t \neq n \in \mathbb{Z}^{+}, t>0
\end{array}
$$

with the impulse conditions

$$
\begin{aligned}
& \triangle x(n)=3^{-n} x(n) \\
& \triangle y(n)=5^{-n} y(n), t=n \in \mathbb{Z}^{+} .
\end{aligned}
$$

It is clear that this satisfies all hypotheses of Theorem 2. So, all solutions of system (3.12)-(3.13) are oscillatory. The solution $\left(x_{n}(t), y_{n}(t)\right)$ of system (3.12)-(3.13) with initial conditions $x(-1)=0.5, x(0)=-10, y(-1)=-0.5, y(0)=0$ is shown in Figure 1.

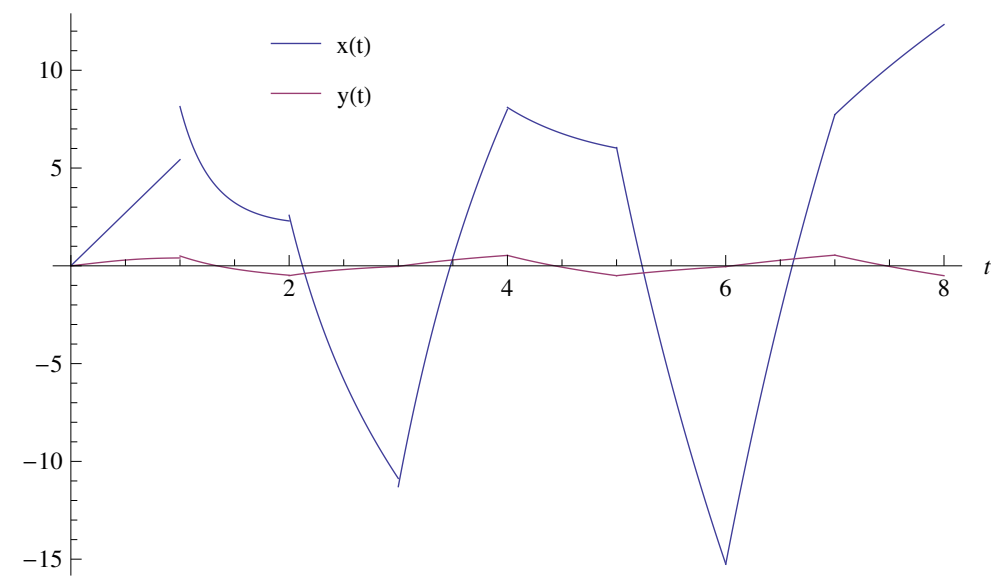

FIGURE 1. Oscillatory solutions of system (3.12)-(3.13) with the initial conditions $x(-1)=0.5, x(0)=-10, y(-1)=-0.5, y(0)=0$.

\section{REFERENCES}

[1] S. Abbas and Y. Xia, "Almost automorphic solutions of impulsive cellular neural networks with piecewise constant argument," Neural Processing Letters, vol. 42, no. 3, pp. 691-702, 2015.

[2] H. Bereketoglu, G. Seyhan, and F. Karakoc, "On a second order differential equation with piecewise constant mixed arguments," Carpathian J. Math., vol. 27, no. 1, pp. 1-12, 2011.

[3] S. Busenberg and K. L. Cooke, "Models of vertically transmitted diseases with sequentialcontinuous dynamics," Nonlinear phenomena in mathematical sciences, Proc. int. Conf., Arlington/Tex. 1980, 179-187 (1982)., 1982.

[4] K.-S. Chiu, "Exponential stability and periodic solutions of impulsive neural network models with piecewise constant argument," Acta Appl. Math., vol. 151, no. 1, pp. 199-226, 2017, doi: 10.1007/s10440-017-0108-3. 
[5] K.-S. Chiu and J.-C. Jeng, "Stability of oscillatory solutions of differential equations with general piecewise constant arguments of mixed type," Math. Nachr., vol. 288, no. 10, pp. 1085-1097, 2015, doi: 10.1002/mana.201300127.

[6] K.-S. Chiu and T. Li, "Oscillatory and periodic solutions of differential equations with piecewise constant generalized mixed arguments," Math. Nachr., vol. 292, no. 10, pp. 2153-2164, 2019, doi: 10.1002/mana.201800053.

[7] K.-S. Chiu, M. Pinto, and J.-C. Jeng, "Existence and global convergence of periodic solutions in recurrent neural network models with a general piecewise alternately advanced and retarded argument," Acta Appl. Math., vol. 133, no. 1, pp. 133-152, 2014, doi: 10.1007/s10440-013-9863y.

[8] L. O. Chua and L. Yang, "Cellular neural networks: Theory," IEEE Transactions on circuits and systems, vol. 35, no. 10, pp. 1257-1272, 1988.

[9] A. Cochocki and R. Unbehauen, Neural networks for optimization and signal processing. John Wiley \& Sons, Inc., 1993.

[10] Z. Huang, X. Wang, and F. Gao, "The existence and global attractivity of almost periodic sequence solution of discrete-time neural networks," Physics Letters A, vol. 350, no. 3-4, pp. 182-191, 2006.

[11] G. Joya, M. A. Atencia, and F. Sandoval, "Hopfield neural networks for optimization: Study of the different dynamics," Neurocomputing, vol. 43, no. 1-4, pp. 219-237, 2002, doi: 10.1016/S09252312(01)00337-X.

[12] F. Karakoc, A. Unal, and H. Bereketoglu, "Oscillation of a nonlinear impulsive differential equation system with piecewise constant argument," Adv. Difference Equ., vol. 2018, p. 11, 2018, id/No 99, doi: 10.1186/s13662-018-1556-4.

[13] W.-J. Li and T. Lee, "Hopfield neural networks for affine invariant matching," IEEE transactions on neural networks, vol. 12, no. 6, pp. 1400-1410, 2001.

[14] M. Pinto and G. Robledo, "Existence and stability of almost periodic solutions in impulsive neural network models," Applied Mathematics and Computation, vol. 217, no. 8, pp. 4167-4177, 2010.

[15] J. Wiener, Generalized solutions of functional differential equations. World Scientific, 1993.

[16] S. S. Young, P. D. Scott, and N. M. Nasrabadi, "Object recognition using multilayer hopfield neural network," IEEE Transactions on Image Processing, vol. 6, no. 3, pp. 357-372, 1997.

Authors' addresses

Mehtap Lafci Büyükkahraman

(corresponding author) Department of Mathematics, Faculty of Arts and Sciences, Uşak University, 64200, Uşak, Turkey

E-mail address: mehtap.lafci@usak.edu.tr

\section{Gizem S. Oztepe}

Department of Mathematics, Faculty of Sciences, Ankara University, 06100, Ankara, Turkey

E-mail address: gseyhan@ankara.edu.tr

\section{Hüseyin Bereketoğlu}

Department of Mathematics, Faculty of Sciences, Ankara University, 06100, Ankara, Turkey

E-mail address: bereketescience.ankara.edu.tr 